Recepción: 20 / 04 / 2017

Aceptación: 20 / 05 / 2017

Publicación: 15 / 07 / 2017

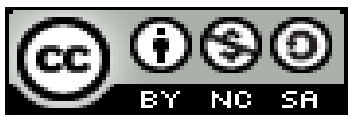

Ciencias de la Salud

Artículo de investigación

\title{
Alargamiento de corona realizado con carillas de composite directas para corregir la desproporción de prótesis parcial metal-porcelana
}

Crown extension made with direct composite veneers to correct the disproportion of partial dentures metal-porcelain

\section{Alongamento da coroa realizada com laminado compósito directa para corrigir a disparidade de d GFP dentadura parcial}

Miguel A. Rodriguez Llaguno ${ }^{\text {I }}$ miguel.rodriguezll@ug.edu.ec

Jorge L. García Villamar ${ }^{\text {II }}$ jorge.garciav@ug.edu.ec

Ernesto R. Montece Seixas III ernesto.monteces@ug.edu.ec

Katherine E. Lima Tamay ${ }^{\text {IV }}$ kat12_1986@hotmail.com

Correspondencia: miguel.rodriguezll@ug.edu.ec

I. Diplomado en Docencia Superior; Magister en Docencia y Gerencia en Educación Superior; Diplomado en Docencia Superior; Doctor en Odontología; Universidad de Guayaquil, Guayaquil, Ecuador.

II. Magister en Diseño Curricular; Diplomado en Docencia Superior; Doctor en Odontología; Universidad de Guayaquil, Guayaquil, Ecuador.

III. Magister en Diseño Curricular; Diploma Superior en Diseño Curricular por Competencias; Diplomado en Docencia Superior; Universidad de Guayaquil, Guayaquil, Ecuador.

IV. Odontóloga; Universidad de Guayaquil, Guayaquil, Ecuador. 


\section{Resumen}

El objetivo del alargamiento de la corona es devolver la estética y armonía del sector anterior superior mediante la confección de carillas de composite utilizando la técnica a mano alzada para corregir la desproporción anatómica de una prótesis parcial fija metal-porcelana. En este caso clínico se describe cómo se puede corregir la estética del sector anterior superior causado por desarmonía entre las piezas dentales naturales y una prótesis parcial fija metal - porcelana de 3 unidades en las piezas 21, 22 y 23. Esto se logra gracias a la realización de carillas de composite en las piezas 11, 12 y 13 mediante las cuales se logró el control del color, morfología y textura, devolviendo la estética a este sector. Se puede concluir que una prótesis parcial fija es una alternativa eficaz para devolver la funcionalidad a un paciente parcialmente edente y es un éxito total cuando cumple los parámetros de estética. Pero cuando no los cumple, provoca una desarmonía que puede ser corregida por medio de carillas de composite. De esta forma se devuelve la estética e influye positivamente en el estado anímico del paciente con un método rápido yeconómico.

Palabras claves: Carilla de composite; prótesis fija; alargamiento de corona. 
Alargamiento de corona realizado con carillas de composite directas para corregir la desproporción de prótesis parcial metalporcelana

\section{Abstract}

The objective of the crown extension is to return the aesthetics and harmony of the upper anterior sector through the preparation of composite veneers using the freehand technique to correct the anatomical disproportion of a fixed metal-porcelain partial denture. In this case, it is described how the aesthetic of the upper anterior sector caused by disharmony between the natural teeth and a fixed partial metal - porcelain prosthesis of 3 units can be corrected in the pieces 21,22 and 23 . This is achieved thanks to the Realization of composite veneers in pieces 11,12 and 13 through which control of color, morphology and texture was achieved, restoring aesthetics to this sector. It can be concluded that a fixed partial denture is an effective alternative to return the functionality to a partially edentulous patient and is a complete success when it complies with the aesthetic parameters. But when it does not comply, it causes a disharmony that can be corrected by means of composite veneers. In this way the aesthetics is returned and positively influences the patient's mood with a fast and economical method.

Key words: Composite veneer; fixed prosthesis; crown elongation. 


\section{Resumo}

O objectivo do alongamento coroa é retornado a estética e a harmonia da anterior superior através da produção de folheados compósitos utilizando a técnica de mão livre para corrigir desproporção anatómica de um GFP dentadura parcial fixa. Neste caso relatório descreve a forma de corrigir a estética do anterior superior causada por desarmonia entre o dente natural e um metal dentadura parcial fixa - 3 unidades de peças de porcelana 21,22 e 23 . Isto é conseguido pela realização de folheados compósitos sobre as partes 11, 12 e 13 através dos quais controlam a cor, textura e morfologia alcançado, recuperando estética deste sector. Pode-se concluir que uma dentadura parcial fixa é eficaz para restaurar a funcionalidade de um paciente edente parcialmente alternativa é inteiramente bem sucedido quando se reúne os parâmetros estéticos. Mas quando isso não acontece, provoca uma desarmonia que pode ser corrigido por folheados compósitos. Assim estética é devolvido e influencia positivamente o estado de espírito do método rápido e económico paciente.

Palavras-chave: Folheado composto; prótese fixa; alongamento da coroa. 
Alargamiento de corona realizado con carillas de composite directas para corregir la desproporción de prótesis parcial metalporcelana

\section{Introducción.}

Para considerar la estética dentofacial, es necesario que las características morfológicas de las piezas dentales cumplan ciertos parámetros (Weber, Fuentes, García, \& Cantín, 2014). La sonrisa expresa muchas emociones en el ser humano y tiene un rol fundamental en la percepción de la belleza (Londoño \& Botero, 2012) (Hernández, Ramos, \& Enríquez, 2015). Los dientes anteriores, en especial los incisivos centrales superiores tienen una gran influencia al momento de percibir la estética de la sonrisa. Las proporciones de tamaño, forma, color de estas piezas deben ser correctas en relación con el resto de la cara (Chiche \& Pinault, 2002) (Batista, Santos, \& Bonfante, 1998) (Korkut, Yanikoglu, \& Gunday, 2013).

Actualmente, existe una gran demanda de tratamientos estéticos para reemplazar dientes que se han perdido en el sector anterior y una buena opción son las prótesis fijas (Figueroa, Cruz, De Carvalho, Leite, \& Cháves, 2014) (Al Baker, Rashid, \& Al Amri, 2016). Las restauraciones de metal cerámica brindan la posibilidad de otorgar un alto grado de estética y funcionalidad a la prótesis fija (Troiano, Sánchez, Benincasa, Haumuller, \& Closas, 2008). El resultado esperado, tanto para el paciente como para el rehabilitador, de una restauración con prótesis fija metal - porcelana es que sea agradable estéticamente (Batista, Santos, \& Bonfante, 1998).

Sin embargo, pueden ocurrir varios errores en la confección de este tipo de restauraciones. Para lograr el éxito, es necesario que, al momento de la preparación del diente, el desgaste sea el suficiente para obtener un espacio con dimensiones adecuadas. Una de las consecuencias de no obtener el espacio necesario es que el laboratorista dental confeccione una corona con el espesor ideal de metal y porcelana para lograr una adecuada resistencia y estética, pero que esto provoca el 
sobrecontorno de la misma, interfiriendo seriamente con las dimensiones normales de las piezas (Batista, Santos, \& Bonfante, 1998).

Para pacientes que presenten problemas estéticos y/o funcionales, las resinas compuestas son una alternativa con un costo y tiempo relativamente bajos (Wahbi, Al Sharief, Tayeb, \& Bokhari, 2012). En los últimos años ha surgido la técnica directa de carillas de resina compuesta. Estas pueden ser colocadas directamente sobre el diente preparado (Korkut, Yanikoglu, \& Gunday, 2013). Las anomalías estéticas de los dientes anteriores como tamaño o color pueden corregidas con este tipo de restauraciones. (Jain, Das, Pruthi, Shah, \& Rajendiran, 2015)

En el caso que se presenta en este trabajo, se busca demostrar que las carillas de resina con técnica directa pueden ser una buena opción para corregir la desproporción de una prótesis fija de metal porcelana, devolviendo de esta forma la estética y bienestar del paciente.

\section{Materiales y métodos.}

\section{Presentación del caso clínico}

Paciente de sexo masculino, de 38 años de edad, asiste a la Facultad Piloto de Odontología de la Universidad de Guayaquil, su motivo de consulta era la falta de la pieza 22 y la queja por la apariencia estética de su sonrisa, debido a la desarmonía del sector anterior superior.

Al examen clínico se observan buenas condiciones de salud bucal. Presenta una prótesis parcial fija desde la pieza 21 hasta la 23. Las pizas 11,12 y 13 presentan desgaste incisal y una morfología que perjudica la estética de este sector. La relación oclusal con los antagonistas inferiores es correcta. 
Alargamiento de corona realizado con carillas de composite directas para corregir la desproporción de prótesis parcial metalporcelana

\section{Diagnóstico.}

Desarmonía en el sector anterior superior.

\section{Plan de tratamiento}

Elaboración de carillas de composite en las piezas 11, 12 y 13.

Figura 1: Estado del paciente antes del tratamiento.

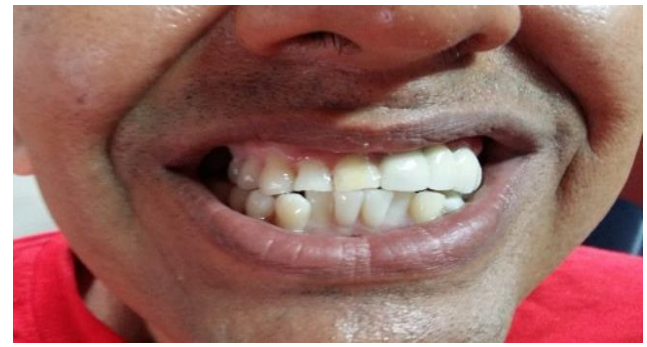

\section{Procedimiento clínico del caso en paciente}

Técnica de preparación de caras vestibulares de las piezas 11,12 y 13.

\section{Reducción de la cara vestibular}

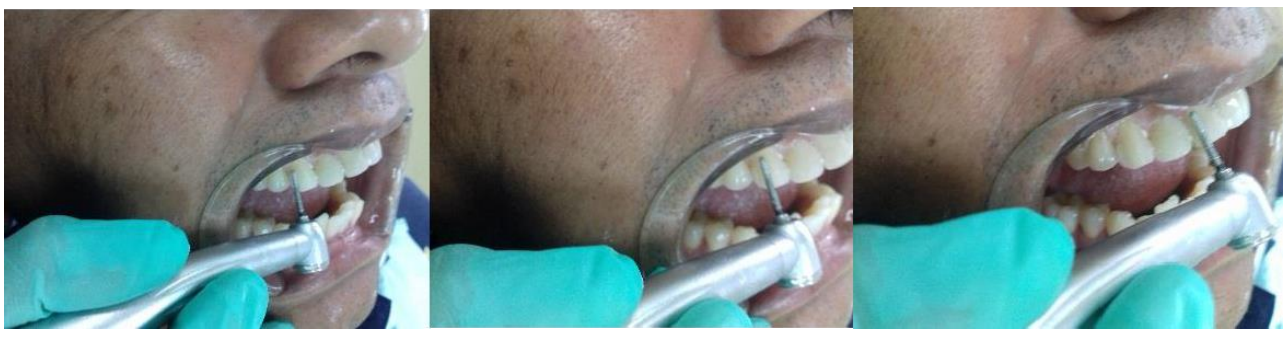

Figura 1: Reducción vestibular de las piezas 11, 12 y 13 con fresa troncocónica punta redonda. 


\section{Reducción cervical}

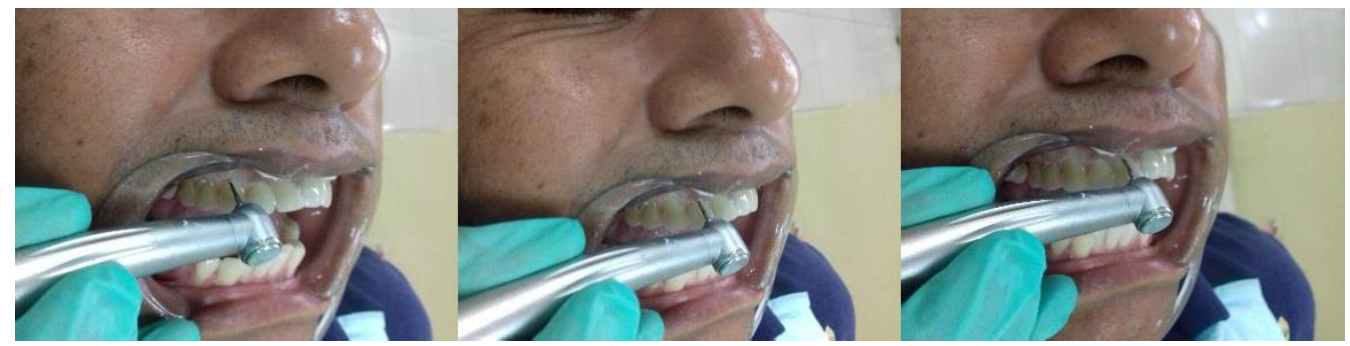

Figura 2: Reducción cervical de las piezas 11, 12 y 13 con fresa redonda pequeña.

\section{Piezas 11, 12 y 13 preparadas}

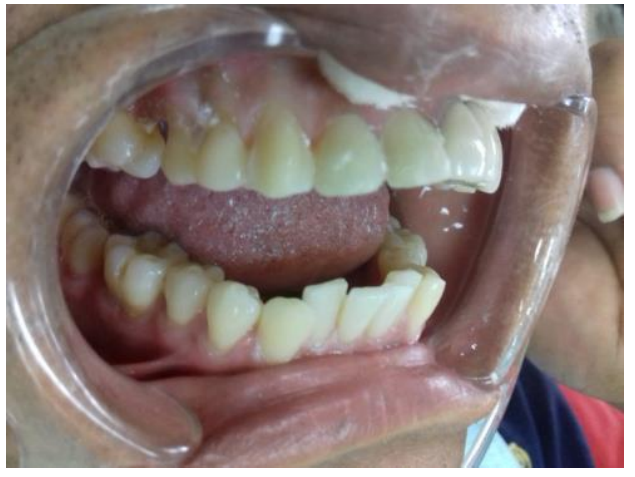

Figura 3: Piezas preparadas.

\section{Aislamiento y protección de piezas vecinas.}

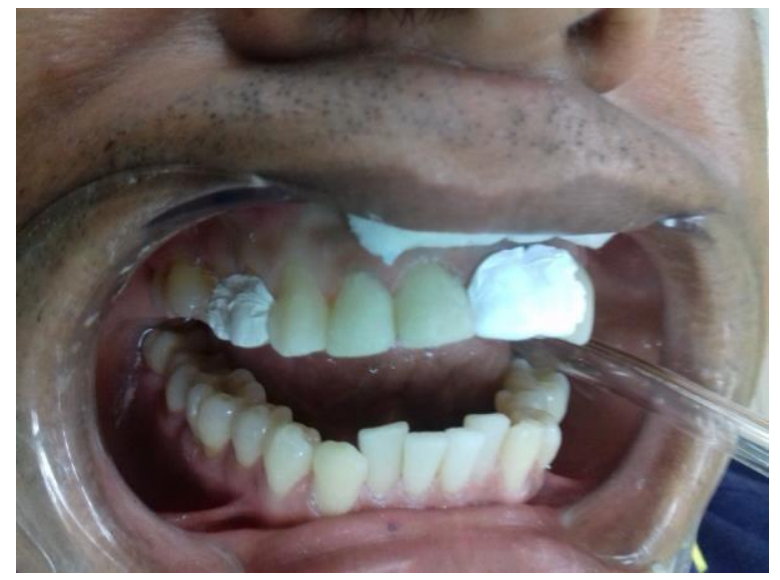

Figura 4: Aislamiento relativo. Protección de piezas vecinas con teflón. 
Alargamiento de corona realizado con carillas de composite directas para corregir la desproporción de prótesis parcial metalporcelana

\section{Acondicionamiento ácido}

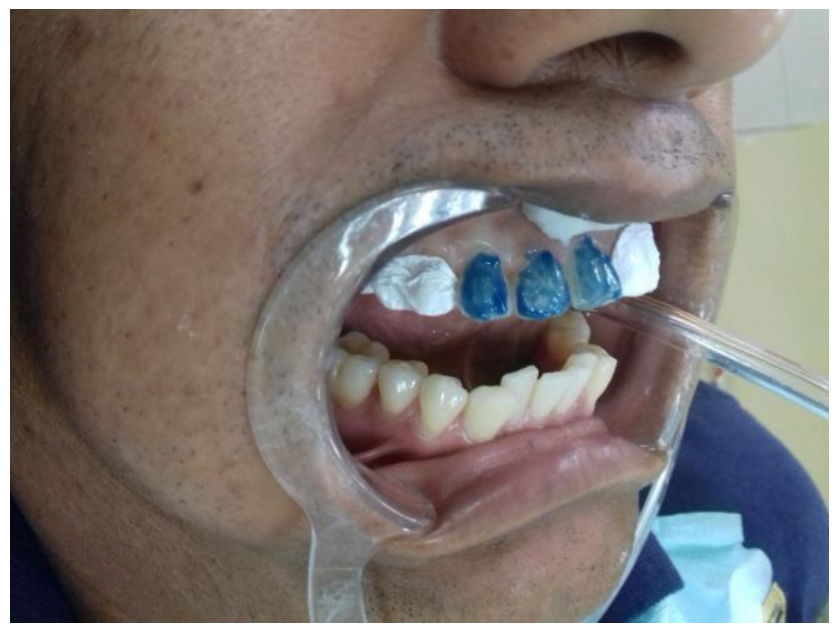

Figura 5: Acondicionamiento de las piezas 11, 12 y 13 con ácido ortofosfórico al 37\%.

\section{Aplicación de bonding}
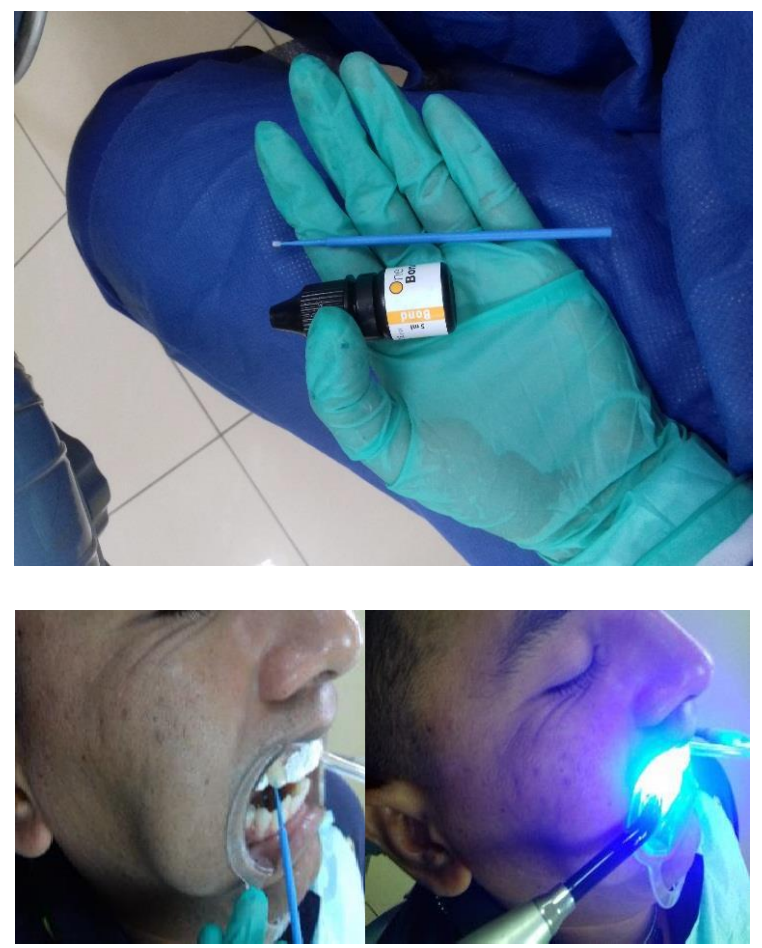

Figura 6: Aplicación de bonding en las piezas previamente lavadas y secadas. Fotopolimerizar por 20 segundos. 


\section{Inserción de resina}

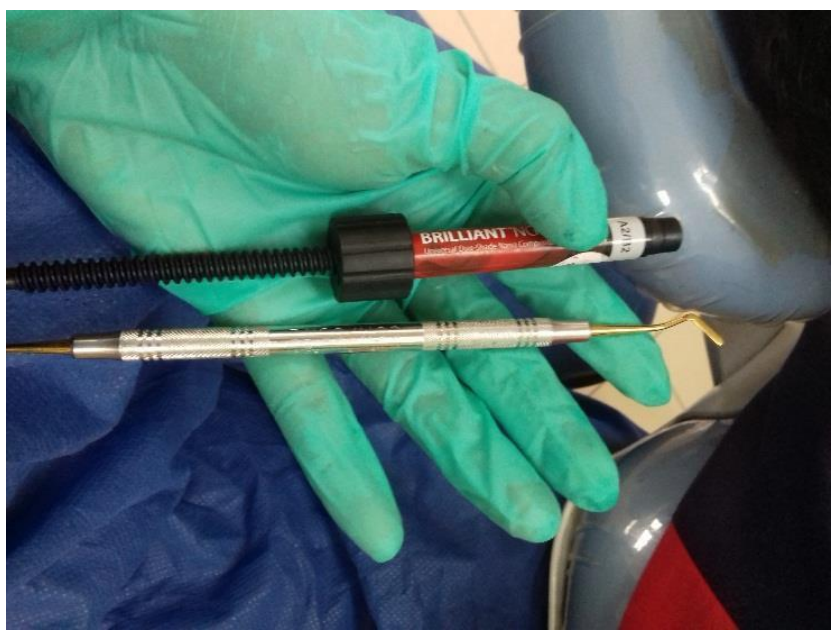

Figura 7: Se utilizó resina nanohíbrida color A2.

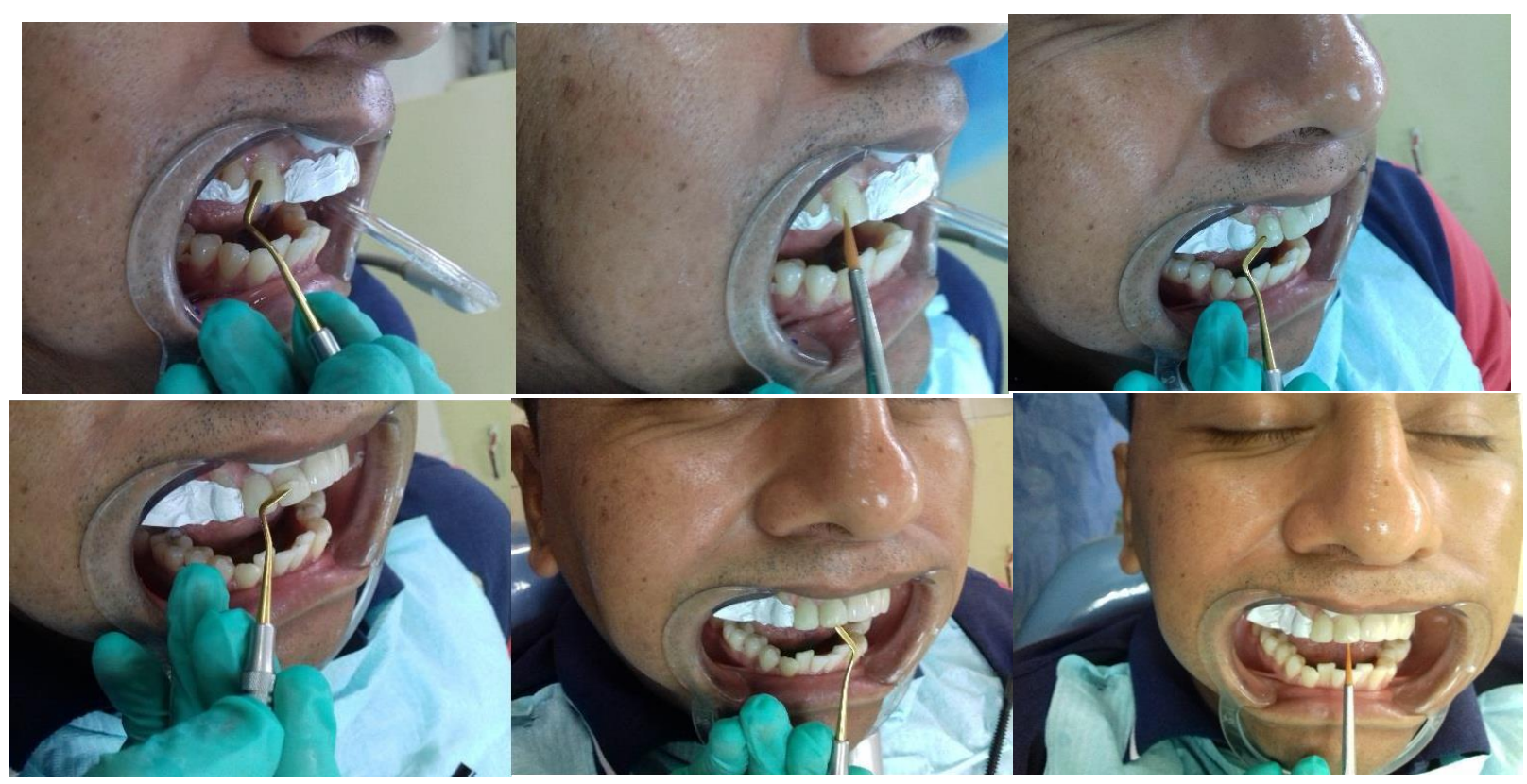

Figura 8: Inserción de resina con ayuda de una espátula en las piezas 11, 12 y 13. Utilización de un pincel "pelo de Martha" para obtener una mejor textura. Por cada incremento de resina se fotopolimeriza 20 segundos. 
Alargamiento de corona realizado con carillas de composite directas para corregir la desproporción de prótesis parcial metalporcelana

\section{Aplicación de glicerina}

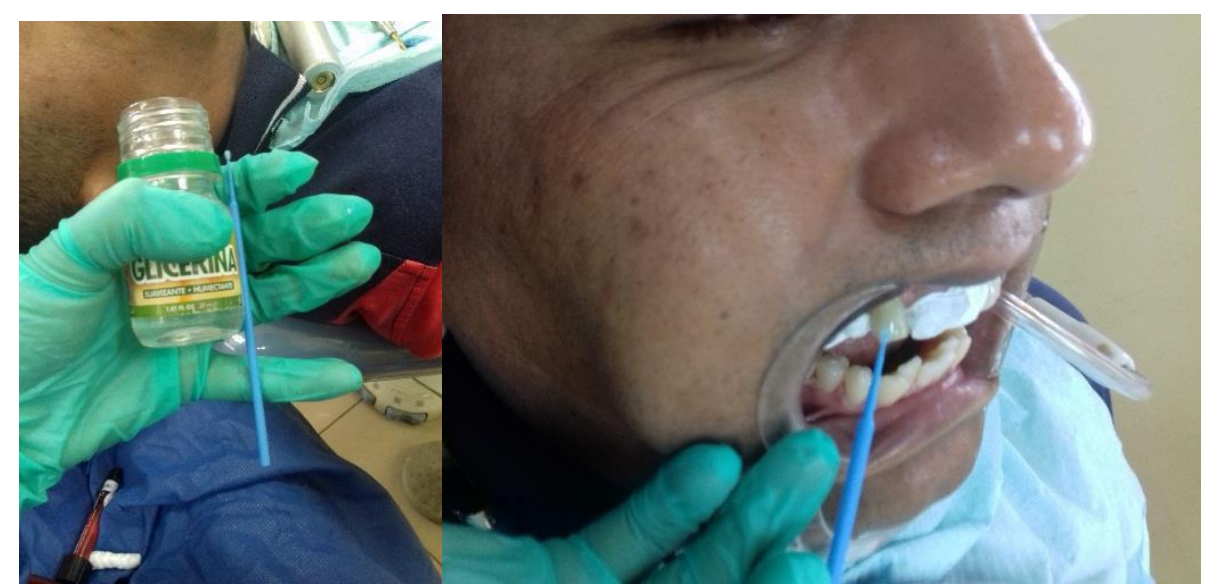

Figura 9: Antes de fotopolimerizar el último incremento de resina, se aplica glicerina para evitar la capa inhibida de oxígeno.

\section{Resultados}

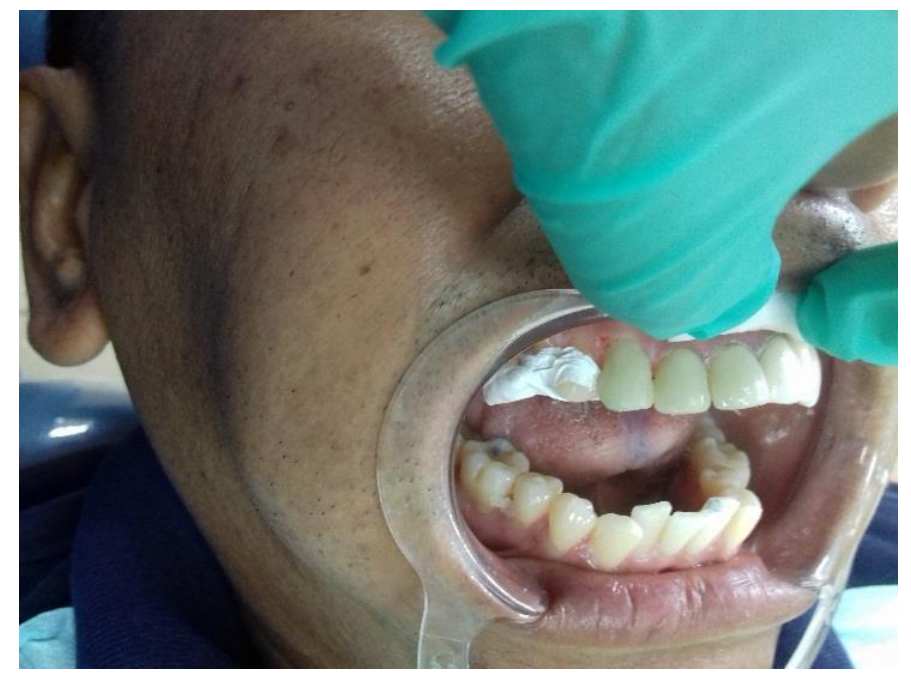

Figura 10: Aspecto de las restauraciones sin pulir. 


\section{Pulido y abrillantado}

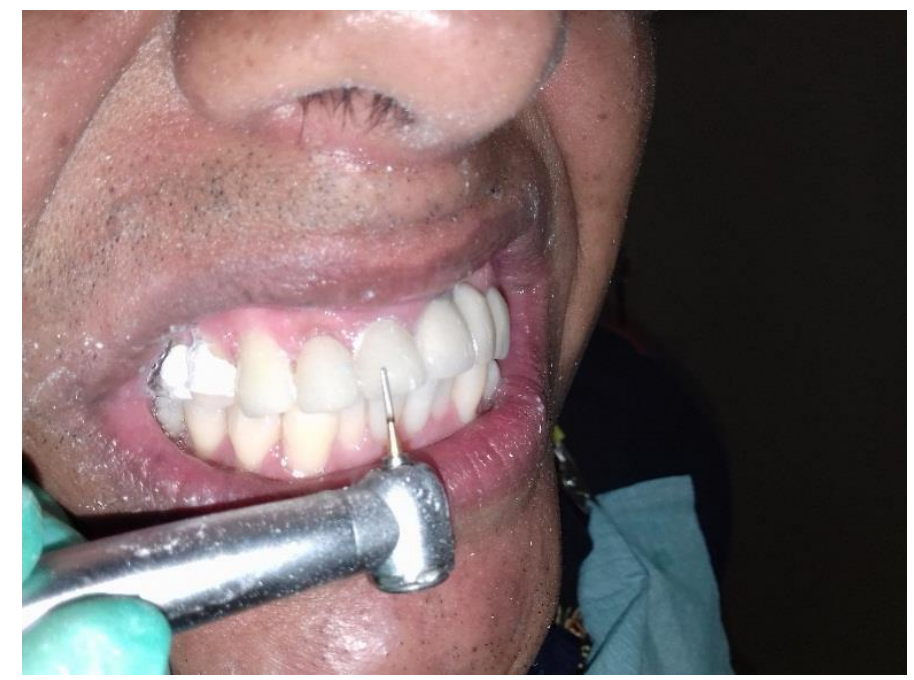

Figura 11: Se pule con una fresa multihojas para retirar excesos de resina.

Carillas terminadas

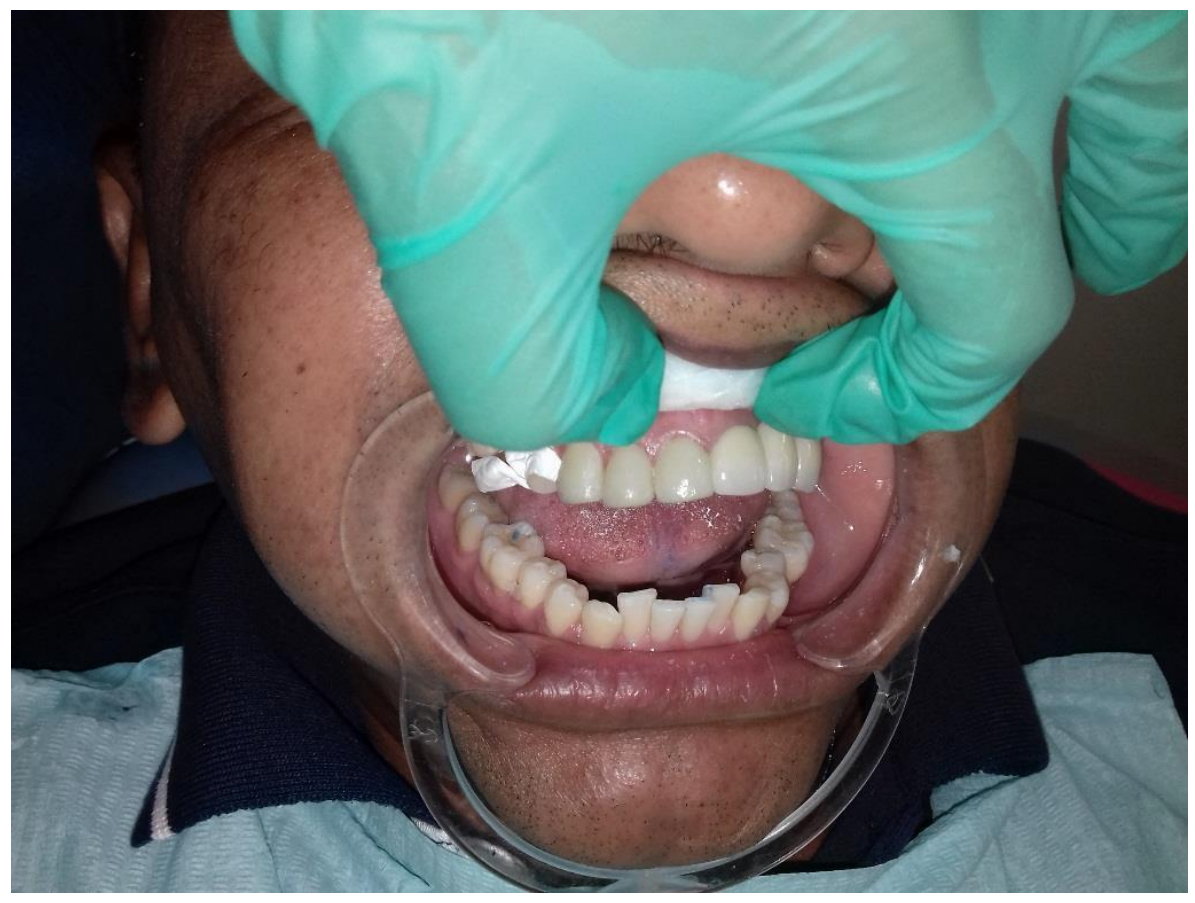

Figura 12: Aspecto de las restauraciones después de pulir. 
Alargamiento de corona realizado con carillas de composite directas para corregir la desproporción de prótesis parcial metalporcelana

\section{Conclusiones.}

La restauración de las piezas perdidas mediante una prótesis parcial fija metal-porcelana en el sector anterior superior devuelve al paciente funcionalidad y estética, siempre que esté en armonía con las piezas naturales del paciente.

Cuando existe una desarmonía entre la prótesis parcial fija y las piezas naturales, una alternativa eficaz, son las carillas de resina. Por medio de este tipo de restauraciones podemos controlar la morfología, color y textura de las piezas. De esta forma podemos armonizar el sector anterior superior con un método rápido, económico y altamente estético.

El resultado final tiene un impacto positivo en el paciente, incluso en su estado psicológico, ya que una sonrisa antiestética provocaba malestar en él.

\section{Bibliografía}

Al Baker, A., Rashid, S., \& Al Amri, M. (2016). Preserving esthetics, occlusion and occlusal vertical dimension in a patient with fixed prostheses seeking dental implant treatment. The Saudi Dental Journal, 203208.

Batista, J., Santos, D., \& Bonfante, G. (1998). Factores estéticos en la preparación del diente para coronas metalocerámicas. Revista Odontológica Dominicana, 182-194.

Chiche, G., \& Pinault, A. (2002). Prótesis fija estética en dientes anteriores. Barcelona: Ed. Masson.

Figueroa, R., Cruz, F., De Carvalho, R., Leite, F., \& Cháves, M. (2014). Rehabilitación de los dientes anteriores con el sistema cerámico disilicato de litio. Int. J. Odontostomat., 469-474.

Hernández, Y., Ramos, D., \& Enríquez, A. (2015). Carillas estéticas con la utilización de resinas compuestas como alternativa ante la hipomineralización. Presentación de un caso. Medisur, 429-435.

Jain, V., Das, T., Pruthi, G., Shah, N., \& Rajendiran, S. (2015). Comparative evaluate of effects of blenching on color stability and marginal adaptation of discolored direct and indirect composite laminate veneers under in vivo conditions. The jorunal of indian prosthodontic society, 46-52.

Korkut, B., Yanikoglu, F., \& Gunday, M. (2013). Direct composite laminte veeners: three case reports. Journal of Dental Research, Dental Clinics, Dental Prospects, 105-111. 
Londoño, M., \& Botero, P. (2012). La sonrisa y sus dimensiones. Rev Fac Odontol Univ Antioq, 353-365.

Troiano, M., Sánchez, P., Benincasa, M., Haumuller, I., \& Closas, J. (2008). Elección del color en la restauración dental. Actas Odontológicas, 46-55.

Wahbi, M., Al Sharief, H., Tayeb, H., \& Bokhari, A. (2012). Minimally invaisve use of coloured composite resin in aesthetic restoration of periodontially involved teeth: case report. The Saudi Dental Journal, 83-89.

Weber, B., Fuentes, R., García, N., \& Cantín, M. (2014). Relaciones de forma y proporción del incisivo central maxilar con medidas faciales, línea mediana dentaria y facial en adultos. Int. J. Morphol, 1101-1107 